\title{
Ulam's stability for some linear conformable fractional differential equations
}

\author{
Sen Wang ${ }^{1}$, Wei Jiang ${ }^{1 *}$, Jiale Sheng ${ }^{1}$ and Rui $\mathrm{Li}^{1}$
}

${ }^{\text {*Correspondence: }}$

jiangwei@ahu.edu.cn

'School of Mathematical Sciences,

Anhui University, Hefei, China

\begin{abstract}
In this paper, by introducing the concepts of Ulam type stability for ODEs into the equations involving conformable fractional derivative, we utilize the technique of conformable fractional Laplace transform to investigate the Ulam-Hyers and Ulam-Hyers-Rassias stability for several kinds of linear differential equations in the frame of conformable fractional derivative.
\end{abstract}

MSC: $34 \mathrm{~A} 08 ; 26 \mathrm{~A} 33$

Keywords: Conformable fractional Laplace transform; Ulam-Hyers and Ulam-Hyers-Rassias stability; Linear differential equations; Conformable fractional derivative

\section{Introduction}

Over the past few decades, the problem of analysis on Ulam type stability (which was first posed by Ulam in [1]) has already been proved to be an important subject in the area of stability theory and mathematical modeling. For details, one can refer to [2-8] and the references therein, where the Ulam type stability of different type equations was discussed via different approaches, like the Gronwall lemma and the method of Picard operators $[2,3]$, the technique of integral factors [4, 5], the Laplace transform approach [6-8].

As we all know, fractional calculus $[9,10]$ is quite a significant branch of mathematical area and has been posed almost at the same time as the classical ones. Fractional calculus has been proved to be a powerful and effective tool for explaining the physical phenomena and has been widely used in fractional modeling which can describe complex systems more specifically and realistically. Many fractional derivatives have been proposed so far, they can be roughly classified into two types: local and nonlocal operators. The nonlocal fractional operators, like the well-known Riemann-Liouville (RL) and Caputo (C) derivatives, the newly defined Atangana-Baleanu-Caputo(ABC) derivative [11] (which generalizes the Caputo-Fabrizio(CF) derivative $[12,13]$ by using the Mittag-Leffler function as the kernel which is both nonsingular and nonlocal), the Caputo-Hadamard $(\mathrm{CH})$ derivative [14, 15], and some generalized nonlocal fractional derivatives [16-19], are very useful for studying the complex dynamical systems in physical phenomena. The unique property that these fractional calculi have in common may be the linearity, while not all these fractional derivatives obey the classical properties like the product rule, the chain rule,

(c) The Author(s) 2020. This article is licensed under a Creative Commons Attribution 4.0 International License, which permits use, sharing, adaptation, distribution and reproduction in any medium or format, as long as you give appropriate credit to the original author(s) and the source, provide a link to the Creative Commons licence, and indicate if changes were made. The images or other third party material in this article are included in the article's Creative Commons licence, unless indicated otherwise in a credit line to the material. If material is not included in the article's Creative Commons licence and your intended use is not permitted by statutory regulation or exceeds the permitted use, you will need to obtain permission directly from the copyright holder. To view a copy of this licence, visit http://creativecommons.org/licenses/by/4.0/. 
etc. In order to overcome these or other difficulties, in [20], the authors introduced a new simple and well-behaved local derivative called conformable fractional derivative, which is just defined on a basic limit definition and satisfies almost all the properties that the classical integer-order derivative owns. In [21], the author developed this new derivative more specifically and generalized many useful and valuable results. Since then, more and more attention [22-28] has been paid to this new derivative, and many questions were solved by using such a definition. Some history about the development of the theory of conformable derivatives can be found in [29,30]. In addition, it is worth noting that the authors in their recent work [31] pointed out that the conformable derivative really results from the ordinary derivative with the change of variable $t \rightarrow(t-a)^{\alpha} / \alpha$.

With the development of fractional calculus, fractional differential equations have been playing an increasingly important role in the field of differential equations, we refer the reader to the papers [32-35], where some new existence results and some new methods of new types of nonlocal fractional operators with nonsingular kernels were discussed. Particularly, the authors in [36] investigated the Hyers-Ulam stability of linear fractional differential equations in the frame of the nonlocal $\mathrm{ABC}$ fractional derivative by using the Laplace transform method (via the Wright function) and established generalized HyersUlam-Rassias stability results for nonlinear problem with the help of Gronwall's inequality. The authors in [19] extended the Ulam-Hyers stability and Ulam-Hyers-Rassias stability theory to differential equations with delay and in the frame of a certain class of generalized Caputo fractional derivatives with dependence on a kernel function, their methods are based on Gronwall's inequality in the frame of the generalized nonlocal fractional operators. The authors in [37] studied the existence of solutions and Ulam's stability for the conformable fractional differential equations with constant coefficients

$$
\left\{\begin{array}{l}
T_{a}^{\alpha} x(t)=\lambda x(t)+f(t, x(t)), t \in(a, b] \text { or }(a, \infty), \quad 0<\alpha<1, \\
x(a)=x_{a},
\end{array}\right.
$$

where $\lambda \in \mathbf{R} /\{0\}, T_{a}^{\alpha} x(t)$ denotes the conformable fractional derivative (CFD) starting from $a$ of a function $x$ of order $\alpha$ (which will be given in Definition 2.1) and $f \in C([a, b] \times \mathbf{R}, \mathbf{R}$ ). After the explicit representation of solutions had been given, their approaches were based upon the Picard iterative approach, the fixed point theories, and the integral inequalities.

Considering the availability and practicability of Laplace transform both in the study of the solutions of integer-order differential equations and fractional-order differential equations, in this paper, we first utilize the method based on the conformable fractional Laplace transform technique (which was introduced in [21]) to investigate Ulam's stability of the following linear fractional differential equation in the frame of the local conformable derivative:

$$
\left\{\begin{array}{l}
T_{t_{0}}^{\alpha} x(t)+\beta x(t)=f(t), t \in\left(t_{0}, T\right), \quad t_{0}<T \leq+\infty, \beta \in \mathbf{R}, \\
x\left(t_{0}\right)=x_{0}
\end{array}\right.
$$

where $t_{0}$ is the initial time of the system, $f$ is continuous on $\left[t_{0},+\infty\right)$ and conformable exponentially bounded (see Definition 2.16). According to [37], we can recall that a function $x \in C^{1}\left(\left[t_{0}, T\right], \mathbf{R}\right)$ is the solution of (1) if $x$ satisfies $T_{t_{0}}^{\alpha} x(t)+\beta x(t)=f(t), t \in\left(t_{0}, T\right]$ and $x\left(t_{0}\right)=x_{0}$. 
Next, this method is also applied to study the Ulam type stability of linear nonhomogenous conformable fractional differential equations, linear Langevin equations described by two same conformable fractional derivatives, and linear conformable integrodifferential equations. Finally, we give an example to illustrate our results.

Notations: $\mathbf{R}^{+}$denotes the set of all real positive numbers, $\mathbf{R}^{n}$ represents an $n$ dimensional space, $\mathbf{R}^{n \times n}$ denotes the space of all $n \times n$ matrices. Let $|x|_{(.)}$be any vector norm (e.g., $\cdot=1,2, \infty)$ and $\|(\cdot)\|$ denote the matrix norm induced by this vector. $C([a, b], \mathbf{R})$ denotes the set of continuous functions from $[a, b]$ into $\mathbf{R}$ and $C^{m}([a, b], \mathbf{R})=$ $\left\{x \in C([a, b], \mathbf{R}): x^{(m)} \in C([a, b], \mathbf{R}\}\right.$, where $x^{(m)}$ denotes the $m$ th continuously differentiable function $x$.

\section{Preliminaries}

This section collects some basic definitions, notations, remark, and necessary lemmas which are used throughout this paper.

Definition 2.1 (see [21] Conformable fractional derivative) Let $f:[a,+\infty) \rightarrow \mathbf{R}$ be a function, then the conformable fractional derivative(CFD) starting from $a$ of the function $f$ of order $\alpha, 0<\alpha \leq 1$, is defined by

$$
T_{a}^{\alpha} f(t)=\lim _{h \rightarrow 0} \frac{f\left(t+h(t-a)^{1-\alpha}\right)-f(t)}{h}
$$

for all $t>a$.

Lemma 2.2 (see [21]) Let $\alpha \in(0,1]$, if $f$ is differentiable, then the following relationship between the CFD and the classical first-order derivative holds:

$$
T_{a}^{\alpha} f(t)=(t-a)^{1-\alpha} f^{\prime}(t)
$$

Definition 2.3 ([21] Conformable factional integral) Let $f:[a,+\infty) \rightarrow \mathbf{R}$ be a function, then the conformable fractional integral(CFI) starting from $a$ of the function $f$ of order $\alpha$, $0<\alpha \leq 1$, is defined by

$$
I_{a}^{\alpha} f(t)=\int_{a}^{t} f(s) d_{\alpha}(s, a)=\int_{a}^{t} f(s)(s-a)^{\alpha-1} d s,
$$

if the Riemann improper integral exists.

Lemma 2.4 (see [21]) Let $\alpha \in(0,1]$, and $f$ be a continuous differentiable function on $[a,+\infty)$, then for all $t>a$, we have

$$
I_{a}^{\alpha} T_{a}^{\alpha} f(t)=f(t)-f(a)
$$

Definition 2.5 (see [21] Conformable fractional exponential function) For every $t \geq a$, the conformable fractional exponential function (CFEF) is defined by

$$
E_{\alpha}(\lambda, t-a)=\exp \left(\lambda \cdot \frac{(t-a)^{\alpha}}{\alpha}\right)=\sum_{k=0}^{\infty} \frac{\lambda^{k}(t-a)^{\alpha k}}{\alpha^{k} k !}, \quad 0<\alpha \leq 1, \lambda \in \mathbf{R} .
$$


Remark 2.6 Like the Mittag-Leffler function's irreplaceable role in the solution of the classical fractional-order differential equations, the conformable fractional exponential function plays an analogous effect in the solution of conformable fractional differential equations.

Lemma 2.7 For any $\beta \in \mathbf{R}$ and $t \geq s \geq a$,

$$
E_{\alpha}(-\beta, t-a) E_{\alpha}(\beta, s-a) \leq 1
$$

Definition 2.8 (see [21] Conformable fractional Laplace transform) Let $\alpha \in(0,1], f$ : $[a,+\infty) \rightarrow \mathbf{R}$ be a real-valued function. Then the conformable fractional Laplace transform (CFLT) starting from $a$ of the function $f$ of order $\alpha$ is defined by

$$
L_{\alpha}\{f(t)\}(s)=\int_{a}^{+\infty} E_{\alpha}(-s, t-a) f(t) d_{\alpha}(t, a)=\int_{a}^{+\infty} E_{\alpha}(-s, t-a) f(t)(t-a)^{\alpha-1} d t .
$$

Lemma 2.9 (see [21]) For a constant $\delta$,

$$
L_{\alpha}\{\delta\}(s)=\frac{\delta}{s}, \quad s>0
$$

Lemma 2.10 (see [21]) Let $f:[a,+\infty) \rightarrow \mathbf{R}$ be a differentiable function such that $L_{\alpha}\{f(t)\}(s)=F_{\alpha}(s)$ exists. Then

$$
L_{\alpha}\left\{T_{a}^{\alpha} f(t)\right\}(s)=s F_{\alpha}(s)-f(a)
$$

and the relationship between the usual Laplace transform and CFLT can be represented as

$$
L_{\alpha}\{f(t)\}(s)=F_{\alpha}(s)=\boldsymbol{L}\left\{f\left(a+(\alpha t)^{1 / \alpha}\right)\right\}(s),
$$

where $\boldsymbol{L}\{f(t)\}=\int_{0}^{+\infty} e^{-s t} f(t) d t$.

Remark 2.11 It is worth mentioning that, unlike the usual Laplace transform, the expression of CFLT demonstrates that it is closely related with the fractional order $\alpha$. In other words, for a function $f$ such that $L_{\alpha}\{f(t)\}(s)$ and $L_{\beta}\{f(t)\}(s)$ exist for $0<\alpha, \beta \leq 1$, then

$$
\alpha \neq \beta \quad \Rightarrow \quad L_{\alpha}\{f(t)\}(s) \neq L_{\beta}\{f(t)\}(s) .
$$

Remark 2.12 Without loss of generality, we can denote $L_{\alpha}^{-1}$ as the inverse of CFLT. For example,

(i) since $L_{\alpha}\left\{E_{\alpha}(\lambda, t-a)\right\}=\boldsymbol{L}\left\{e^{\lambda t}\right\}=\frac{1}{s-\lambda}, s>\lambda$, we have

$$
L_{\alpha}^{-1}\left\{\frac{1}{s-\lambda}\right\}=E_{\alpha}(\lambda, t-a)
$$

(ii) due to $L_{\alpha}\left\{1-E_{\alpha}(-\lambda, t-a)\right\}=\boldsymbol{L}\left\{1-e^{-\lambda t}\right\}=\frac{\lambda}{s(s+\lambda)}, s>0$, then

$$
L_{\alpha}^{-1}\left\{\frac{\lambda}{s(s+\lambda)}\right\}=1-E_{\alpha}(-\lambda, t-a)
$$


(iii) since $L_{\alpha}\left\{\cos \omega \frac{(t-a)^{\alpha}}{\alpha}\right\}=\boldsymbol{L}\{\cos \omega t\}=\frac{s}{\omega^{2}+s^{2}}, s>0$, then

$$
L_{\alpha}^{-1}\left\{\frac{s}{\omega^{2}+s^{2}}\right\}=\cos \omega \frac{(t-a)^{\alpha}}{\alpha}
$$

Definition 2.13 (see [25] Conformable convolution of two functions) Let $f, g:[a,+\infty) \rightarrow$ $\mathbf{R}$ be two real-valued functions such that $f$ is the function of $(t-a)^{\alpha}$ for $0<\alpha \leq 1$, then the conformable convolution of these two functions is defined by

$$
(f * g)(t)=\int_{a}^{t} f\left((t-a)^{\alpha}-(\theta-a)^{\alpha}\right) g(\theta)(\theta-a)^{\alpha-1} d \theta .
$$

Lemma 2.14 (see [25] Conformable convolution theorem) Under the above conformable convolution definition, if $L_{\alpha}\{f\}(s)$ and $L_{\alpha}\{g\}(s)$ exist, then

$$
L_{\alpha}\{f * g\}=L_{\alpha}\{f\} \cdot L_{\alpha}\{g\}
$$

Remark 2.15 In view of the definition of CFI and conformable convolution, it follows that

$$
I_{a}^{\alpha} f(t)=\int_{a}^{t} f(s)(s-a)^{\alpha-1} d s=1 * f(t)
$$

and hence,

$$
L_{\alpha}^{-1}\left\{I_{a}^{\alpha} f(t)\right\}=L_{\alpha}^{-1}\{1 * f(t)\}=\frac{L_{\alpha}\{f\}}{s}, \quad s>0 .
$$

Definition 2.16 (see [22] Conformable exponential bounded function) For a function $f$ defined on $[a,+\infty) \rightarrow \mathbf{R}$, if there exist positive real constants $M, c$, and $0<\alpha \leq 1$ such that

$$
|f(t)| \leq M E_{\alpha}(c, t-a)
$$

holds for all sufficiently large $t$, then we call $f$ conformable exponential bounded.

Remark 2.17 Considering Eq. (1) and referring to [18], for a continuous function $f$ defined on $\left[t_{0},+\infty\right) \rightarrow \mathbf{R}$, if $f$ is conformable exponential bounded, then $x(t)$ and $T_{t_{0}}^{\alpha} x(t)$ are both conformable exponential bounded, and thus, $L_{\alpha}\{x(t)\}(s)$ and $L_{\alpha}\left\{T_{t_{0}}^{\alpha} x(t)\right\}(s)$ all exist.

Lemma 2.18 (see [21]) Assume that $f \in C^{2}([a,+\infty), \mathbf{R})$ and $0<\alpha \leq \frac{1}{2}$, then

$$
T_{a}^{\alpha} T_{a}^{\alpha} f(x)= \begin{cases}(1-\alpha)(x-a)^{1-2 \alpha} f^{\prime}(x)+(x-a)^{2-2 \alpha} f^{\prime \prime}(x), & x>a, \\ 0, & x=a .\end{cases}
$$

\section{Main results}

In this section, we utilize the technique of CFLT to investigate the Ulam type stability of the linear conformable fractional differential equation (1). 
Definition 3.1 Eq. (1) is said to be Ulam-Hyers stable if there exists a constant $\gamma>0$ such that, for each $\varepsilon>0$ and for each solution $y$ of the inequality

$$
\left|T_{t_{0}}^{\alpha} y(t)+\beta y(t)-f(t)\right| \leq \varepsilon, \quad t \in J:=\left[t_{0}, T\right)
$$

there exists a solution $x$ of Eq. (1) with

$$
|y(t)-x(t)| \leq \gamma \varepsilon, \quad t \in J
$$

Remark 3.2 The existence and uniqueness results of the solutions for initial value problem (1) have been studied in considerable detail (see [23, 37]). In addition, it can be derived from $[21,23,25]$ that the general solution of (1) is given by

$$
x(t)=E_{\alpha}\left(-\beta, t-t_{0}\right) x_{0}+\int_{t_{0}}^{t} E_{\alpha}\left(-\beta, t-t_{0}\right) E_{\alpha}\left(\beta, \tau-t_{0}\right)\left(\tau-t_{0}\right)^{\alpha-1} f(\tau) d \tau .
$$

Theorem 3.3 If a function $y: J \rightarrow \mathbf{R}$ satisfies inequality (2) and for some $\varepsilon>0$, then there exists a solution $x$ of Eq. (1) such that

$$
|y(t)-x(t)| \leq \frac{\left(t-t_{0}\right)^{\alpha}}{\alpha} \varepsilon, \quad \forall t \in J .
$$

Proof Let

$$
z(t)=T_{t_{0}}^{\alpha} y(t)+\beta y(t)-f(t), \quad t \in J
$$

By taking CFLT of (5), it can be deduced that

$$
L_{\alpha}\{z\}=s L_{\alpha}\{y\}-y\left(t_{0}\right)+\beta L_{\alpha}\{y\}-L_{\alpha}\{f\} .
$$

Further, it follows that

$$
L_{\alpha}\{y\}=\frac{y\left(t_{0}\right)+L_{\alpha}\{f\}}{s+\beta}+\frac{L_{\alpha}\{z\}}{s+\beta} .
$$

If we set

$$
x(t)=E_{\alpha}\left(-\beta, t-t_{0}\right) y\left(t_{0}\right)+E_{\alpha}\left(-\beta, t-t_{0}\right) * f(t),
$$

then, clearly, $x(t)$ is differentiable on $\left(t_{0}, T\right)$, and

$$
x\left(t_{0}\right)=y\left(t_{0}\right)=x_{0} .
$$

Taking CFLT on both sides of (7) yields

$$
L_{\alpha}\{x\}=\frac{y\left(t_{0}\right)+L_{\alpha}\{f\}}{s+\beta},
$$


which is equivalent to

$$
s L_{\alpha}\{x\}-x\left(t_{0}\right)+\beta L_{\alpha}\{x\}=L_{\alpha}\left\{T_{t_{0}}^{\alpha} x+\beta x\right\}=L_{\alpha}\{f\} .
$$

The one-to-one property of the operator $L_{\alpha}$ guarantees that

$$
T_{t_{0}}^{\alpha} x(t)+\beta x(t)=f(t)
$$

Thus, the function $x$ is a solution of Eq. (1). Combining (6) and (8) and noting that

$$
L_{\alpha}\left\{E_{\alpha}\left(-\beta, t-t_{0}\right) * z\right\}=L_{\alpha}\left\{E_{\alpha}\left(-\beta, t-t_{0}\right)\right\} \cdot L_{\alpha}\{z(t)\}=\frac{L_{\alpha}\{z\}}{s+\beta},
$$

we get

$$
L_{\alpha}\{y\}-L_{\alpha}\{x\}=\frac{L_{\alpha}\{z\}}{s+\beta}=L_{\alpha}\left\{E_{\alpha}\left(-\beta, t-t_{0}\right) * z\right\},
$$

which shows that

$$
y(t)-x(t)=E_{\alpha}\left(-\beta, t-t_{0}\right) * z(t), \quad t \in J .
$$

In view of (2), it holds that $|z(t)| \leq \varepsilon, t \in J$. Therefore,

$$
\begin{aligned}
|y(t)-x(t)| & =\left|E_{\alpha}\left(-\beta, t-t_{0}\right) * z(t)\right| \\
& =\left|\int_{t_{0}}^{t} E_{\alpha}\left(-\beta, t-t_{0}\right) E_{\alpha}\left(\beta, \tau-t_{0}\right)\left(\tau-t_{0}\right)^{\alpha-1} z(\tau) d \tau\right| \\
& \leq \int_{t_{0}}^{t}\left(\tau-t_{0}\right)^{\alpha-1}\left|E_{\alpha}\left(-\beta, t-t_{0}\right) E_{\alpha}\left(\beta, \tau-t_{0}\right) z(\tau)\right| d \tau \\
& \leq \varepsilon \int_{t_{0}}^{t}\left(\tau-t_{0}\right)^{\alpha-1}\left|E_{\alpha}\left(-\beta, t-t_{0}\right) E_{\alpha}\left(\beta, \tau-t_{0}\right)\right| d \tau \\
& \leq \varepsilon \frac{\left(t-t_{0}\right)^{\alpha}}{\alpha},
\end{aligned}
$$

where Lemma 2.7 is used. Hence the proof is completed.

Remark 3.4 According to Theorem 3.3, we note that if $T<+\infty$, then (1) is Ulam-Hyers stable on $J$ with $\gamma=\frac{\left(T-t_{0}\right)^{\alpha}}{\alpha}$. If $T=+\infty$, then (1) is not Ulam-Hyers stable.

Definition 3.5 Eq. (1) is said to be Ulam-Hyers-Rassias stable if there exists a constant $\gamma^{*}>0$ such that, for each $\varepsilon>0$ and for each solution $y$ of the inequality

$$
\left|T_{t_{0}}^{\alpha} y(t)+\beta y(t)-f(t)\right| \leq \varepsilon h(t), \quad t \in J,
$$

there exists a solution $x$ of Eq. (1) with

$$
|y(t)-x(t)| \leq \gamma^{*} \operatorname{ch}(t), \quad t \in J,
$$

where $h \in C\left(J, \mathbf{R}^{+}\right)$is a positive function. 
Next, proceeding as in the proof of Theorem 3.3, we give the following result.

Theorem 3.6 If the function $y: J \rightarrow \mathbf{R}$ satisfies inequality (10) and for some $\varepsilon>0$, then Eq. (1) is Ulam-Hyers-Rassias stable provided that

$$
\int_{t_{0}}^{t}(\tau-a)^{\alpha-1} h(\tau) d \tau=I_{t_{0}}^{\alpha} h(t) \leq c_{h} h(t), \quad c_{h} \in \mathbf{R}^{+} .
$$

Proof The proof is completely similar to Theorem 3.3, we only need to note that, under assumption (12), it can follow that

$$
\begin{aligned}
|y(t)-x(t)| & =\left|E_{\alpha}\left(-\beta, t-t_{0}\right) * z(t)\right| \\
& \leq \int_{t_{0}}^{t}\left(\tau-t_{0}\right)^{\alpha-1}\left|E_{\alpha}\left(-\beta, t-t_{0}\right) E_{\alpha}\left(\beta, \tau-t_{0}\right) z(\tau)\right| d \tau \\
& \leq \varepsilon \int_{t_{0}}^{t}\left(\tau-t_{0}\right)^{\alpha-1}\left|E_{\alpha}\left(-\beta, t-t_{0}\right) E_{\alpha}\left(\beta, \tau-t_{0}\right)\right| h(\tau) d \tau \\
& \leq \varepsilon \int_{t_{0}}^{t}\left(\tau-t_{0}\right)^{\alpha-1} h(\tau) d \tau=\varepsilon I_{t_{0}}^{\alpha} h(t) \leq \varepsilon c_{h} h(t),
\end{aligned}
$$

thus, (11) is satisfied, and Eq. (1) is Ulam-Hyers-Rassias stable with $\gamma^{*}=c_{h}$.

\section{Further outstretched results}

In this section, by using the methods in the above section, we further investigate Ulam's stability of the following three different type linear differential equations involving CFD.

(i) Linear non-homogenous conformable fractional differential equation

$$
\left\{\begin{array}{l}
T_{t_{0}}^{\alpha} x(t)+A x(t)=f(t), \quad t \in\left(t_{0}, T\right], T<+\infty \\
x\left(t_{0}\right)=x_{0}
\end{array}\right.
$$

where $f \in C\left(\left[t_{0},+\infty\right), \mathbf{R}^{n}\right)$ is conformable exponential bounded, $A \in \mathbf{R}^{n \times n}$.

By applying the CFLT, the solution of (13) is given by (see [28])

$$
x(t)=E_{\alpha}\left(-A, t-t_{0}\right) x_{0}+\int_{t_{0}}^{t} E_{\alpha}\left(-A, t-t_{0}\right) E_{\alpha}\left(A, \tau-t_{0}\right)\left(\tau-t_{0}\right)^{\alpha-1} f(\tau) d \tau .
$$

Let $J:=\left[t_{0}, T\right], \varepsilon>0$, and $h \in C\left(J, \mathbf{R}^{+}\right)$. Consider Eq. (13) and the inequalities

$$
\left\|T_{t_{0}}^{\alpha} x(t)+A x(t)-f(t)\right\| \leq \varepsilon, \quad t \in J,
$$

and

$$
\left\|T_{t_{0}}^{\alpha} x(t)+A x(t)-f(t)\right\| \leq \varepsilon h(t), \quad t \in J .
$$

Definition 4.1 Eq. (13) is said to be Ulam-Hyers stable if there exists a constant $\sigma>0$ such that, for each $\varepsilon>0$ and for each solution $y \in C\left(J, \mathbf{R}^{n}\right)$ of inequality (15), there exists a solution $x \in C^{1}\left(J, \mathbf{R}^{n}\right)$ of Eq. (13) with

$$
\|y(t)-x(t)\| \leq \sigma \varepsilon, \quad t \in J .
$$


Definition 4.2 Eq. (13) is said to be Ulam-Hyers-Rassias stable if there exists a constant $\sigma^{*}>0$ such that, for each $\varepsilon>0$ and for each solution $y \in C\left(J, \mathbf{R}^{n}\right)$ of inequality (16), there exists a solution $x \in C^{1}\left(J, \mathbf{R}^{n}\right)$ of Eq. (13) with

$$
\|y(t)-x(t)\| \leq \sigma^{*} \operatorname{\varepsilon h}(t), \quad t \in J .
$$

Lemma 4.3 For a matrix $A \in \mathbf{R}^{n \times n}, t_{0} \leq s \leq t \leq T<+\infty$, we have

$$
\left\|E_{\alpha}\left(-A, t-t_{0}\right)\right\| \leq E_{\alpha}\left(\|-A\|, t-t_{0}\right)
$$

and

$$
\left\|E_{\alpha}\left(-A, t-t_{0}\right) \cdot E_{\alpha}\left(A, s-t_{0}\right)\right\| \leq\left\|E_{\alpha}\left(-A, t-t_{0}\right)\right\| \cdot\left\|E_{\alpha}\left(A, s-t_{0}\right)\right\| .
$$

Proof The proof can be easily derived from the basic facts in matrix theory that

$$
\left\|e^{A}\right\| \leq e^{\|A\|}
$$

and

$$
\|A B\| \leq\|A\|\|B\|,
$$

respectively, where $B \in \mathbf{R}^{n \times n}$.

Remark 4.4 Denote the matrix function as $E_{1}(t):=E_{\alpha}\left(-A, t-t_{0}\right), E_{2}(t):=E_{\alpha}\left(A, t-t_{0}\right)$, and $M:=\sup _{t_{0} \leq t \leq T}\left\|E_{1}(t)\right\|, N:=\sup _{t_{0} \leq t \leq T}\left\|E_{2}(t)\right\|$. Then

$$
\left\|E_{1}(t)\right\| \leq M, \quad\left\|E_{2}(t)\right\| \leq N
$$

and

$$
\left\|E_{\alpha}\left(-A, t-t_{0}\right) \cdot E_{\alpha}\left(A, s-t_{0}\right)\right\| \leq M N .
$$

Theorem 4.5 If a function $y: J \rightarrow \mathbf{R}^{n}$ satisfies inequality (15) and for some $\varepsilon>0$, then Eq. (13) is Ulam-Hyers stable with $\sigma=\frac{M N\left(T-t_{0}\right)^{\alpha}}{\alpha}$.

Proof Due to the similarity to the proof of Theorem 3.3, we only build the framework here.

Let

$$
z(t)=T_{t_{0}}^{\alpha} y(t)+A y(t)-f(t), \quad t \in J .
$$

Then it follows that $\|z(t)\| \leq \varepsilon$ owing to (15). We can obtain

$$
\begin{aligned}
\|y(t)-x(t)\| & =\left\|E_{\alpha}\left(-A, t-t_{0}\right) * z(t)\right\| \\
& \leq \int_{t_{0}}^{t}\left(\tau-t_{0}\right)^{\alpha-1}\left\|E_{\alpha}\left(-A, t-t_{0}\right) E_{\alpha}\left(A, \tau-t_{0}\right) z(\tau)\right\| d \tau
\end{aligned}
$$




$$
\begin{aligned}
& \leq \varepsilon \int_{t_{0}}^{t}\left(\tau-t_{0}\right)^{\alpha-1}\left\|E_{\alpha}\left(-A, t-t_{0}\right) E_{\alpha}\left(A, \tau-t_{0}\right)\right\| d \tau \\
& \leq \varepsilon \frac{M N\left(t-t_{0}\right)^{\alpha}}{\alpha} \leq \varepsilon \frac{M N\left(T-t_{0}\right)^{\alpha}}{\alpha}
\end{aligned}
$$

where Lemma 4.3 and Remark 4.4 are utilized and $x(t)=E_{\alpha}\left(-A, t-t_{0}\right) y\left(t_{0}\right)+E_{\alpha}(-A, t-$ $\left.t_{0}\right) * f(t)$. That is to say, there exists a solution $x \in C^{1}\left(\left[t_{0}, T\right], \mathbf{R}^{n}\right)$ of Eq. (13) such that (17) holds with $\sigma=\frac{M N\left(T-t_{0}\right)^{\alpha}}{\alpha}$.

Theorem 4.6 Consider Eq. (13) and the inequality

$$
\left\|T_{t_{0}}^{\alpha} y(t)+A y(t)-f(t)\right\| \leq \varepsilon h(t), \quad t \in J .
$$

Then Eq. (13) is Ulam-Hyers-Rassias stable if (12) holds true, that is,

$$
\|y(t)-x(t)\| \leq \varepsilon \sigma^{*} h(t), \quad t \in J
$$

here $\sigma^{*}=c_{h} M N, c_{h}>0$.

(ii) Linear Langevin equations with two same conformable fractional derivatives

$$
\left\{\begin{array}{l}
T_{t_{0}}^{\alpha}\left(T_{t_{0}}^{\alpha}+\lambda\right) x(t)=f(t), \quad t \in\left(t_{0}, T\right], T<+\infty, \lambda>0, \\
x\left(t_{0}\right)=x_{0}
\end{array}\right.
$$

where $0<\alpha \leq \frac{1}{2}, f$ is continuous on $\left[t_{0},+\infty\right)$ and conformable exponentially bounded. In view of Lemma 2.18, we give the concept of a solution for (19).

Definition 4.7 A function $x \in C^{2}\left(\left[t_{0}, T\right], \mathbf{R}\right)$ is a solution of Eq. (19) if $x$ satisfies $T_{t_{0}}^{\alpha}\left(T_{t_{0}}^{\alpha}+\right.$ $\lambda) x(t)=f(t), t \in\left(t_{0}, T\right]$ and $x\left(t_{0}\right)=x_{0}$.

We first derive the representation of the solution of Eq. (19). According to Remark 3.2, we know that the solution of the equation

$$
\left(T_{t_{0}}^{\alpha}+\lambda\right) x(t)=g(t), \quad t \in\left(t_{0}, T\right], x\left(t_{0}\right)=x_{0}, \lambda>0,
$$

can be expressed as

$$
x(t)=E_{\alpha}\left(-\lambda, t-t_{0}\right) x_{0}+\int_{t_{0}}^{t} E_{\alpha}\left(-\lambda, t-t_{0}\right) E_{\alpha}\left(\lambda, \tau-t_{0}\right)\left(\tau-t_{0}\right)^{\alpha-1} g(\tau) d \tau .
$$

Taking the integral operator $I_{t_{0}}^{\alpha}$ on Eq. (19) from $t_{0}$ to $t$ and using Lemma 2.4, we have

$$
\left(T_{t_{0}}^{\alpha}+\lambda\right) x(t)-\left(T_{t_{0}}^{\alpha}+\lambda\right) x\left(t_{0}\right)=I_{t_{0}}^{\alpha} f(t)=\int_{t_{0}}^{t}\left(s-t_{0}\right)^{\alpha-1} f(s) d s
$$

Noticing that the CFD of a constant is zero (see [20,21]), hence $T_{t_{0}}^{\alpha}\left[x\left(t_{0}\right)\right]=0$, then (21) becomes

$$
\left(T_{t_{0}}^{\alpha}+\lambda\right) x(t)=\lambda x_{0}+\int_{t_{0}}^{\tau}\left(s-t_{0}\right)^{\alpha-1} f(s) d s
$$


In addition, note that

$$
\left(\tau-t_{0}\right)^{\alpha-1} E_{\alpha}\left(-\lambda, t-t_{0}\right) E_{\alpha}\left(\lambda, \tau-t_{0}\right)=\frac{d}{d \tau}\left[\frac{1}{\lambda} E_{\alpha}\left(-\lambda, t-t_{0}\right) E_{\alpha}\left(\lambda, \tau-t_{0}\right)\right],
$$

which implies that

$$
\int_{t_{0}}^{t}\left(\tau-t_{0}\right)^{\alpha-1} E_{\alpha}\left(-\lambda, t-t_{0}\right) E_{\alpha}\left(\lambda, \tau-t_{0}\right) d \tau=\frac{1}{\lambda}\left(1-E_{\alpha}\left(-\lambda, t-t_{0}\right)\right) .
$$

Combining (20), (22) with (23), then the final formula of the solution of (19) should be

$$
\begin{aligned}
x(t)= & E_{\alpha}\left(-\lambda, t-t_{0}\right) x_{0}+\int_{t_{0}}^{t} E_{\alpha}\left(-\lambda, t-t_{0}\right) E_{\alpha}\left(\lambda, \tau-t_{0}\right)\left(\tau-t_{0}\right)^{\alpha-1} \\
& \times\left[\lambda x_{0}+\int_{t_{0}}^{\tau}\left(s-t_{0}\right)^{\alpha-1} f(s) d s\right] d \tau \\
= & E_{\alpha}\left(-\lambda, t-t_{0}\right) x_{0}+\lambda x_{0} \cdot \frac{1}{\lambda}\left(1-E_{\alpha}\left(-\lambda, t-t_{0}\right)\right) \\
& +\int_{t_{0}}^{t} \int_{t_{0}}^{\tau}\left(s-t_{0}\right)^{\alpha-1}\left(\tau-t_{0}\right)^{\alpha-1} E_{\alpha}\left(-\lambda, t-t_{0}\right) E_{\alpha}\left(\lambda, \tau-t_{0}\right) f(s) d s d \tau \\
= & x_{0}+\int_{t_{0}}^{t} \int_{t_{0}}^{\tau}\left(s-t_{0}\right)^{\alpha-1}\left(\tau-t_{0}\right)^{\alpha-1} E_{\alpha}\left(-\lambda, t-t_{0}\right) E_{\alpha}\left(\lambda, \tau-t_{0}\right) f(s) d s d \tau \\
= & x_{0}+\int_{t_{0}}^{t} \int_{s}^{t}\left(s-t_{0}\right)^{\alpha-1}\left(\tau-t_{0}\right)^{\alpha-1} E_{\alpha}\left(-\lambda, t-t_{0}\right) E_{\alpha}\left(\lambda, \tau-t_{0}\right) f(s) d \tau d s \\
= & x_{0}+\int_{t_{0}}^{t}\left(s-t_{0}\right)^{\alpha-1}\left(1-E_{\alpha}\left(-\lambda, t-t_{0}\right) E_{\alpha}\left(\lambda, s-t_{0}\right)\right) f(s) d s \\
= & x_{0}+\frac{1}{\lambda}\left(1-E_{\alpha}\left(-\lambda, t-t_{0}\right)\right) * f(t) .
\end{aligned}
$$

Next, we consider Eq. (19) and the inequalities

$$
\left|T_{t_{0}}^{\alpha}\left(T_{t_{0}}^{\alpha}+\lambda\right) x(t)-f(t)\right| \leq \varepsilon, \quad t \in J
$$

and

$$
\left|T_{t_{0}}^{\alpha}\left(T_{t_{0}}^{\alpha}+\lambda\right) x(t)-f(t)\right| \leq \varepsilon h(t), \quad t \in J, h \in C\left(J, \mathbf{R}^{+}\right) .
$$

Definition 4.8 Eq. (19) is said to be Ulam-Hyers stable if there exists a constant $\kappa>0$ such that, for each $\varepsilon>0$ and for each solution $y$ of inequality (24), there exists a solution $x$ of Eq. (19) with

$$
|y(t)-x(t)| \leq \kappa \varepsilon, \quad t \in J .
$$

Definition 4.9 Eq. (19) is said to be Ulam-Hyers-Rassias stable if there exists a constant $\kappa^{*}>0$ such that, for each $\varepsilon>0$ and for each solution $y$ of inequality (25), there exists a solution $x$ of Eq. (19) with

$$
|y(t)-x(t)| \leq \kappa^{*} \varepsilon h(t), \quad t \in J .
$$


Theorem 4.10 If a function $y: J \rightarrow \mathbf{R}$ satisfies inequality (24) and for some $\varepsilon>0$, then Eq. (19) is Ulam-Hyers stable with $\kappa=\frac{\left(T-t_{0}\right)^{\alpha}}{\lambda \alpha}$.

Proof Let

$$
z(t)=T_{t_{0}}^{\alpha}\left(T_{t_{0}}^{\alpha}+\lambda\right) y(t)-f(t), \quad t \in J
$$

Taking the CFLT on the above equation, we have

$$
L_{\alpha}\{z\}=\left(s^{2}+\lambda s\right) L_{\alpha}\{y\}-(s+\lambda) y\left(t_{0}\right)-L_{\alpha}\{f\} .
$$

Then

$$
L_{\alpha}\{y\}=\frac{y\left(t_{0}\right)}{s}+\frac{L_{\alpha}\{f\}}{s(s+\lambda)}+\frac{L_{\alpha}\{z\}}{s(s+\lambda)} .
$$

Define the function $x$ as

$$
x(t)=y\left(t_{0}\right)+\frac{1}{\lambda}\left(1-E_{\alpha}\left(-\lambda, t-t_{0}\right)\right) * f(t),
$$

then $x(t)$ is secondly differentiable on $\left(t_{0}, T\right]$ and

$$
x\left(t_{0}\right)=y\left(t_{0}\right)=x_{0} .
$$

An application of CFLT to (29) yields that

$$
L_{\alpha}\{x\}=L_{\alpha}\left\{y\left(t_{0}\right)\right\}+L_{\alpha}\left\{\frac{1}{\lambda}\left(1-E_{\alpha}\left(-\lambda, t-t_{0}\right)\right) * f(t)\right\}=\frac{x\left(t_{0}\right)}{s}+\frac{L_{\alpha}\{f\}}{s(s+\lambda)},
$$

which implies that

$$
s(s+\lambda) L_{\alpha}\{x\}-(s+\lambda) x\left(t_{0}\right)=L_{\alpha}\left\{T_{t_{0}}^{\alpha}\left(T_{t_{0}}^{\alpha}+\lambda\right) x\right\}=L_{\alpha}\{f\} .
$$

Since $L_{\alpha}$ is one-to-one, it follows that

$$
T_{t_{0}}^{\alpha}\left(T_{t_{0}}^{\alpha}+\lambda\right) x(t)=f(t)
$$

namely, $x$ is a solution of Eq. (19). Noting that

$$
L_{\alpha}\left\{\frac{1}{\lambda}\left(1-E_{\alpha}\left(-\lambda, t-t_{0}\right)\right) * z\right\}=L_{\alpha}\left\{\frac{1}{\lambda}\left(1-E_{\alpha}\left(-\lambda, t-t_{0}\right)\right)\right\} \cdot L_{\alpha}\{z\}=\frac{L_{\alpha}\{z\}}{s(s+\lambda)},
$$

and combining (28) with (30), we obtain

$$
L_{\alpha}\{y\}-L_{\alpha}\{x\}=\frac{L_{\alpha}\{z\}}{s(s+\lambda)}=L_{\alpha}\left\{\frac{1}{\lambda}\left(1-E_{\alpha}\left(-\lambda, t-t_{0}\right)\right) * z\right\},
$$

hence

$$
y(t)-x(t)=\frac{1}{\lambda}\left(1-E_{\alpha}\left(-\lambda, t-t_{0}\right)\right) * z(t), \quad t \in J .
$$


According to (24), it holds that $|z(t)| \leq \varepsilon, t \in J$. Therefore,

$$
\begin{aligned}
|y(t)-x(t)| & =\frac{1}{\lambda}\left|\left(1-E_{\alpha}\left(-\lambda, t-t_{0}\right)\right) * z(t)\right| \\
& =\frac{1}{\lambda}\left|\int_{t_{0}}^{t}\left[1-e^{-\lambda\left(\frac{\left(t-t_{0}\right)^{\alpha}}{\alpha}-\frac{\left(s-t_{0}\right)^{\alpha}}{\alpha}\right)}\right]\left(s-t_{0}\right)^{\alpha-1} z(s) d s\right| \\
& \leq \varepsilon \frac{\left(t-t_{0}\right)^{\alpha}}{\lambda \alpha} \leq \varepsilon \frac{\left(T-t_{0}\right)^{\alpha}}{\lambda \alpha},
\end{aligned}
$$

where the basic fact that $\left|1-e^{-\lambda\left(\frac{\left(t-t_{0}\right)^{\alpha}}{\alpha}-\frac{\left(s-t_{0}\right)^{\alpha}}{\alpha}\right)}\right|<1$ is considered. Then we conclude that there exists a solution $x \in C^{2}\left(\left[t_{0}, T\right], \mathbf{R}\right)$ of Eq. (19) such that (26) holds with $\kappa=\frac{\left(T-t_{0}\right)^{\alpha}}{\lambda \alpha}$. Hence the proof is completed.

Theorem 4.11 Consider Eq. (19) and the inequality

$$
\left|T_{t_{0}}^{\alpha}\left(T_{t_{0}}^{\alpha}+\lambda\right) y(t)-f(t)\right| \leq \varepsilon h(t), \quad t \in J
$$

Then Eq. (19) is Ulam-Hyers-Rassias stable with $\kappa^{*}=\frac{c_{h}}{\lambda}$ provided that (12) holds, that is,

$$
|y(t)-x(t)| \leq \varepsilon \frac{c_{h}}{\lambda} h(t), \quad t \in J
$$

(iii) Linear conformable integro-differential equation

$$
\left\{\begin{array}{l}
T_{t_{0}}^{\alpha} x(t)+I_{t_{0}}^{\alpha} x(t)=f(t), \quad t \in J^{\prime}:=\left(t_{0}, T\right], T<+\infty \\
x\left(t_{0}\right)=x_{0}
\end{array}\right.
$$

where $0<\alpha \leq 1, J:=\left[t_{0}, T\right]$, the function $f: J \rightarrow \mathbf{R}$ is continuous, conformable exponentially bounded and satisfies $f\left(t_{0}\right) \equiv 0$. Clearly, a function $x \in C^{1}(J, \mathbf{R})$ is called the solution of $(31)$ if and only if $x$ satisfies $T_{t_{0}}^{\alpha} x(t)+I_{t_{0}}^{\alpha} x(t)=f(t), t \in J^{\prime}$ with the initial condition $x\left(t_{0}\right)=x_{0}$. We first apply CFLT to seek the solution of Eq. (31).

Taking the CFLT on both sides of Eq. (31) yields that

$$
s L_{\alpha}\{x\}-x_{0}+\frac{1}{S} L_{\alpha}\{x\}=L_{\alpha}\{f\}
$$

which is equivalent to

$$
L_{\alpha}\{x\}=\frac{s}{s^{2}+1} x_{0}+\frac{s}{s^{2}+1} L_{\alpha}\{f\}
$$

Using the inverse CFLT and noting Remark 2.15, we obtain the solution

$$
x(t)=\cos \frac{\left(t-t_{0}\right)^{\alpha}}{\alpha} x_{0}+\cos \frac{\left(t-t_{0}\right)^{\alpha}}{\alpha} * f(t) .
$$

Now, we utilize the same method to investigate Ulam type stability of Eq. (31). Consider the inequalities

$$
\left|T_{t_{0}}^{\alpha} x(t)+I_{t_{0}}^{\alpha} x(t)-f(t)\right| \leq \varepsilon, \quad t \in J
$$


and

$$
\left|T_{t_{0}}^{\alpha} x(t)+I_{t_{0}}^{\alpha} x(t)-f(t)\right| \leq \varepsilon h(t), \quad t \in J, h \in C(J, \mathbf{R}) .
$$

Definition 4.12 Eq. (31) is said to be Ulam-Hyers stable if there exists a constant $\rho>0$ such that, for each $\varepsilon>0$ and for each solution $y$ of inequality (33), there exists a solution $x$ of Eq. (31) with

$$
|y(t)-x(t)| \leq \rho \varepsilon, \quad t \in J .
$$

Definition 4.13 Eq. (31) is said to be Ulam-Hyers-Rassias stable if there exists a constant $\rho^{*}>0$ such that, for each $\varepsilon>0$ and for each solution $y$ of inequality (34), there exists a solution $x$ of Eq. (31) with

$$
|y(t)-x(t)| \leq \rho^{*} \varepsilon h(t), \quad t \in J
$$

Theorem 4.14 If a function $y: J \rightarrow \mathbf{R}$ satisfies inequality (33) and for some $\varepsilon>0$, then Eq. (31) is Ulam-Hyers stable with $\rho=\frac{\left(T-t_{0}\right)^{\alpha}}{\alpha}$.

Proof Let

$$
z(t)=T_{t_{0}}^{\alpha} y(t)+I_{t_{0}}^{\alpha} y(t)-f(t), \quad t \in J
$$

An application of CFLT to (37) yields that

$$
L_{\alpha}\{z\}=\left(s+\frac{1}{s}\right) L_{\alpha}\{y\}-y\left(t_{0}\right)-L_{\alpha}\{f\},
$$

which indicates that

$$
L_{\alpha}\{y\}=\frac{s}{s^{2}+1} y\left(t_{0}\right)+\frac{s}{s^{2}+1} L_{\alpha}\{f\}+\frac{s}{s^{2}+1} L_{\alpha}\{z\} .
$$

Set the function $x$ as

$$
x(t)=\cos \frac{\left(t-t_{0}\right)^{\alpha}}{\alpha} y\left(t_{0}\right)+\cos \frac{\left(t-t_{0}\right)^{\alpha}}{\alpha} * f(t),
$$

then $x(t)$ is differentiable on $\left(t_{0}, T\right]$ with $x\left(t_{0}\right)=y\left(t_{0}\right)=x_{0}$. Taking CFLT on (39), we have

$$
L_{\alpha}\{x\}=\frac{s}{s^{2}+1} y\left(t_{0}\right)+\frac{s}{s^{2}+1} L_{\alpha}\{f\}
$$

which is equivalent to

$$
\left(s+\frac{1}{s}\right) L_{\alpha}\{x\}-x\left(t_{0}\right)=L_{\alpha}\left\{T_{t_{0}}^{\alpha} x(t)+I_{t_{0}}^{\alpha} x(t)\right\}=L_{\alpha}\{f\} .
$$

Therefore, $T_{t_{0}}^{\alpha} x(t)+I_{t_{0}}^{\alpha} x(t)=f(t)$ holds for $\left(t_{0}, T\right]$ with $x\left(t_{0}\right)=x_{0}$, namely, such a function $x$ is a solution of Eq. (31). In addition, let us combine (38) with (40) and note that

$$
L_{\alpha}\left\{\cos \frac{\left(t-t_{0}\right)^{\alpha}}{\alpha} * z\right\}=\frac{s}{s^{2}+1} L_{\alpha}\{z\},
$$


it can be easily deduced that

$$
L_{\alpha}\{y\}-L_{\alpha}\{x\}=\frac{s}{s^{2}+1} L_{\alpha}\{z\}=L_{\alpha}\left\{\cos \frac{\left(t-t_{0}\right)^{\alpha}}{\alpha} * z\right\},
$$

this implies that

$$
y(t)-x(t)=\cos \frac{\left(t-t_{0}\right)^{\alpha}}{\alpha} * z(t) .
$$

Consider (33), it satisfies $|z(t)| \leq \varepsilon, t \in J$. Thus,

$$
\begin{aligned}
|y(t)-x(t)| & =\left|\cos \frac{\left(t-t_{0}\right)^{\alpha}}{\alpha} * z(t)\right| \\
& =\left|\int_{t_{0}}^{t}\left(s-t_{0}\right)^{\alpha-1} \cos \frac{\left(t-t_{0}\right)^{\alpha}-\left(s-t_{0}\right)^{\alpha}}{\alpha} z(s) d s\right| \\
& \leq \varepsilon \int_{t_{0}}^{t}\left(s-t_{0}\right)^{\alpha-1}\left|\cos \frac{\left(t-t_{0}\right)^{\alpha}-\left(s-t_{0}\right)^{\alpha}}{\alpha}\right| d s \\
& \leq \varepsilon \frac{\left(T-t_{0}\right)^{\alpha}}{\alpha} .
\end{aligned}
$$

Hence, there exists a solution $x$ of Eq. (31) such that (35) holds with $\rho=\frac{\left(T-t_{0}\right)^{\alpha}}{\alpha}$.

Theorem 4.15 Consider Eq. (31) and the inequality

$$
\left|T_{t_{0}}^{\alpha} y(t)+I_{t_{0}}^{\alpha} y(t)-f(t)\right| \leq \varepsilon h(t), \quad t \in J .
$$

Then Eq. (31) is Ulam-Hyers-Rassias stable with $\rho^{*}=c_{h}$ provided that (12) holds, that is,

$$
|y(t)-x(t)| \leq \varepsilon c_{h} h(t), \quad t \in J .
$$

\section{An illustrative example}

In this section, an example is given to demonstrate our results.

Let $\alpha=\beta=\frac{1}{2}, t_{0}=0, T=1, f(t)=\sqrt{t} e^{-\sqrt{t}}, x(0)=1$, then $\gamma=\frac{\left(T-t_{0}\right)^{\alpha}}{\alpha}=2$, and Eq. (1) becomes

$$
\left\{\begin{array}{l}
T_{0}^{\frac{1}{2}} x(t)+\frac{1}{2} x(t)=\sqrt{t} e^{-\sqrt{t}}, \quad t \in(0,1], \\
x(0)=1 .
\end{array}\right.
$$

Consider

$$
\left|T_{0}^{\frac{1}{2}} x(t)+\frac{1}{2} x(t)-\sqrt{t} e^{-\sqrt{t}}\right| \leq \varepsilon, \quad t \in[0,1]
$$

and

$$
\left|T_{0}^{\frac{1}{2}} x(t)+\frac{1}{2} x(t)-\sqrt{t} e^{-\sqrt{t}}\right| \leq \operatorname{ch}(t), \quad t \in[0,1] .
$$


According to Definition 3.1 and Theorem 3.3, for some given $\varepsilon>0$ and corresponding solution $y$ such that inequality (43) holds, if we find a solution $x$ of (42) such that $\mid y(t)-$ $x(t) \mid \leq 2 \varepsilon$ holds for all $t \in[0,1]$, then (42) is Ulam-Hyers stable on the interval $[0,1]$.

Choose $\varepsilon=\sqrt{3}$ and set the function $y$ as

$$
y(t)=(2 \sqrt{t}+1) e^{-\sqrt{t}}
$$

then $y(0)=x(0)=1$, and elementary computation yields that

$$
\left|T_{0}^{\frac{1}{2}} y(t)+\frac{1}{2} y(t)-\sqrt{t} e^{-\sqrt{t}}\right|=\left|(1-\sqrt{t}) e^{-\sqrt{t}}\right| \leq \max _{t \in[0,1]}\left|(1-\sqrt{t}) e^{-\sqrt{t}}\right|=1<\sqrt{3},
$$

hence, (43) holds. In addition, following the proof of Theorem 3.3, we can set the function $x$ as

$$
x(t)=E_{\frac{1}{2}}\left(-\frac{1}{2}, t\right) \times 1+E_{\frac{1}{2}}\left(-\frac{1}{2}, t\right) *\left(\sqrt{t} e^{-\sqrt{t}}\right)=(t+1) e^{-\sqrt{t}} .
$$

One can easily note that such an $x$ is a solution of (42), and

$$
|y(t)-x(t)|=\left|(2 \sqrt{t}-t) e^{-\sqrt{t}}\right| \leq \max _{t \in[0,1]}\left|(2 \sqrt{t}-t) e^{-\sqrt{t}}\right| \approx 0.466<2 \sqrt{3}
$$

Moreover, set the function $h$ as $h(t)=\sqrt{t} e^{t}+1$ and let $\gamma^{*}=c_{h}=\pi+1$, then we obtain

$$
I_{t_{0}}^{\alpha} h(t)=e^{t}+2 \sqrt{t}-1 \leq e+1 \leq(\pi+1)\left(\sqrt{t} e^{t}+1\right)=c_{h} h(t)
$$

which implies that (12) holds. Further, obviously,

$$
\left|T_{0}^{\frac{1}{2}} y(t)+\frac{1}{2} y(t)-\sqrt{t} e^{-\sqrt{t}}\right|=\left|(1-\sqrt{t}) e^{-\sqrt{t}}\right| \leq 1 \leq \sqrt{3}\left(\sqrt{t} e^{t}+1\right)=\varepsilon h(t), \quad t \in[0,1]
$$

and

$$
|y(t)-x(t)|=\left|(2 \sqrt{t}-t) e^{-\sqrt{t}}\right|<0.5 \leq \sqrt{3}(\pi+1)\left(\sqrt{t} e^{t}+1\right)=\gamma^{*} \varepsilon h(t) .
$$

From the above discussion, we can conclude that Eq. (42) is Ulam-Hyers stable with $\gamma=2$ and Ulam-Hyers-Rassias stable with $\gamma^{*}=\pi+1$.

\section{Acknowledgements}

The authors acknowledge the support by the National Natural Science Foundation of China (11601003; 11371027;

11471015).

Funding

This work is supported by the National Natural Science Foundation of China $(11601003 ; 11371027 ; 11471015)$

Availability of data and materials

No data were used to support this study.

Competing interests

The authors declare that they have no competing interests.

Authors' contributions

The authors contributed equally to the manuscript. Both of them read and approved the final manuscript. 


\section{Publisher's Note}

Springer Nature remains neutral with regard to jurisdictional claims in published maps and institutional affiliations.

\section{Received: 12 February 2020 Accepted: 29 April 2020 Published online: 28 May 2020}

\section{References}

1. Ulam, S.M.: A Collection of Mathematical Problems. Interscience Tracts in Pure and Applied Mathematics, vol. 8 (1960)

2. Rus, I.A.: Remarks on Ulam stability of the operatorial equations. Fixed Point Theory 10, 305-320 (2009)

3. Rus, I.A.: Ulam stability of ordinary differential equations. Stud. Univ. Babeş-Bolyai, Math. 54, 125-134 (2009)

4. Li, Y., Chen, Y.: Hyers-Ulam stability of linear differential equations of second order. Appl. Math. Lett. 23, 306-309 (2010)

5. Wang, G., Zhou, M., Sun, L.: Hyers-Ulam stability of linear differential equations of first order. Appl. Math. Lett. 21, 1024-1028 (2008)

6. Rezaei, H., Jung, S.M., Rassias, T.M.: Laplace transform and Hyers-Ulam stability of linear differential equations. J. Math. Anal. Appl. 403, 244-251 (2013)

7. Wang, J., Li, X.Z:: A uniform method to Ulam-Hyers stability for some linear fractional equations. Mediterr. J. Math. 13, 625-635 (2016)

8. Alqifiary, Q.H., Jung, S.M.: Laplace transform and generalized Hyers-Ulam stability of linear differential equations. Electron. J. Differ. Equ. 2014, 80 (2014)

9. Podlubny, I.: Fractional Differential Equations. Academic Press, San Diego (1999)

10. Kilbas, A.A., Srivastava, H.M., Trujillo, J.J.: Theory and Applications of Fractional Differential Equations. North-Holland Mathematics Studies., vol. 204. Elsevier, Amsterdam (2006)

11. Atangana, A., Balenau, D.: New fractional derivatives with non-local and non-singular kernel theory and application to heat transfer model. Therm. Sci. 20(2), 763-769 (2016)

12. Caputo, M., Fabrizio, M.: A new definition of fractional derivative without singular kernel. Prog. Fract. Differ. Appl. 2, 73-85 (2015)

13. Atangana, A.: On the new fractional derivative and application to nonlinear Fisher's reaction-diffusion equation. Appl. Math. Comput. 273, 948-956 (2016)

14. Jarad, F., Abdeljawad, T., Baleanu, D.: Caputo-type modification of the Hadamard fractional derivatives. Adv. Differ. Equ. 2012, 142 (2012)

15. Gambo, Y.Y., Jarad, F., Baleanu, D., Abdeljawad, T.: On Caputo modification of the Hadamard fractional derivatives. Adv. Differ. Equ. 2014, 10 (2014)

16. Jarad, F., Uğurlu, E., Abdeljawad, T., Baleanu, D.: On a new class of fractional operators. Adv. Differ. Equ. 2017, 247 (2017)

17. Gambo, Y.Y., Ameen, R., Jarad, F., Abdeljawad, T.: Existence and uniqueness of solutions to fractional differential equations in the frame of generalized Caputo fractional derivatives. Adv. Differ. Equ. 2018, 134 (2018)

18. Adjabi, Y., Jarad, F., Abdeljawad, T.: On generalized fractional operators and a Gronwall type inequality with applications. Filomat 31(17), 5457-5473 (2017)

19. Ameen, R., Jarad, F., Abdeljawad, T.: Ulam stability for delay fractional differential equations with a generalized Caputo derivative. Filomat 32(15), 5265-5274 (2018)

20. Khalil, R., Horani, M.A., Yousef, A., Sababheh, M.: A new definition of fractional derivative. J. Comput. Appl. Math. 264, 65-70 (2014)

21. Abdeljawad, T.: On conformable fractional calculus. J. Comput. Appl. Math. 279, 57-66 (2015)

22. Fernando, S.S., Makhlouf, A.B., Mohamed, A.H.: Conformable Laplace transform of fractional differential equations. Axioms 7, 55 (2018)

23. Benaoumeur, B., Torres, D.F.M.: Existence of solution to a local nonlinear differential equation. J. Comput. Appl. Math. $312,127-133(2017)$

24. Abdourazek, S., Makhlouf, A.B., Mohamed, A.H.: Stability analysis of conformable fractional-order nonlinear systems. Indag. Math. 28, 1265-1274 (2017)

25. Eroglu, B.I, Avci, D., Ozdemir, N.: Optimal control problem for a conformable fractional heat conduction equation. Acta Phys. Pol. A 132, 658-662 (2017)

26. Chung, W.S.: Fractional Newton mechanics with conformable fractional derivative. J. Comput. Appl. Math. 290, 150-158 (2015)

27. Hammad, M.A., Khalil, R.: Abel's formula and Wronskian for conformable fractional differential equations. Int. J. Differ. Equ. Appl. 13(3), 177-183 (2014)

28. Eslami, M., Rezazadeh, H.: The first integral method for Wu-Zhang system with conformable time-fractional derivative. Calcolo 53(3), 475-485 (2016)

29. Abdeljawad, T., Alzabut, J., Jarad, F.: A generalized Lyapunov-type inequality in the frame of conformable derivatives. Adv. Differ. Equ. 2017, 321 (2017)

30. Al-Rifae, M., Abdeljawad, T.: Fundamental results of conformable Sturm-Liouville eigenvalue problems. Complexity 2017, Article ID 3720471 (2017)

31. Anderson, D.R., Camrud, E., Ulness, D.J.: On the nature of the conformable derivative and its applications to physics, J. Fract. Calc. Appl. 10(2), 92-135 (2019)

32. Panda, S.K., Abdeljawad, T., Ravichandran, C.: A complex valued approach to the solutions of Riemann-Liouville integral, Atangana-Baleanu integral operator and non-linear telegraph equation via fixed point method. Chaos Solitons Fractals 2020, 130 (2020)

33. Panda, S.K., Abdeljawad, T., Ravichandran, C.: Novel fixed point approach to Atangana-Baleanu fractional and $L^{p}$-Fredholm integral equations. Alex. Eng. J. (2020). https://doi.org/10.1016/j.aej.2019.12.027

34. Alqudah, M.A., Ravichandran, C., Abdeljawad, T., Valliammal, N.: New results on Caputo fractional-order neutral differential inclusions without compactness. Adv. Differ. Equ. 2019, 528 (2019)

35. Ravichandran, C., Logeswari, K., Jarad, F.: New results on existence in the framework of Atangana-Baleanu derivative for fractional integro-differential equations. Chaos Solitons Fractals 125, 194-200 (2020) 
36. Liu, K., Wang, J., Zhou, Y., O'Regan, D.: Hyers-Ulam stability and existence of solutions for fractional differential equations with Mittag-Leffler kernel. Chaos Solitons Fractals 2020, 132 (2020)

37. Li, M.M., Wang, J., O'Regan, D.: Existence and Ulam's stability for conformable fractional differential equations with constant coefficients. Bull. Malays. Math. Sci. Soc. 42, 1791-1812 (2019)

Submit your manuscript to a SpringerOpen ${ }^{\circ}$ journal and benefit from:

- Convenient online submission

- Rigorous peer review

- Open access: articles freely available online

- High visibility within the field

- Retaining the copyright to your article

Submit your next manuscript at $\boldsymbol{\nabla}$ springeropen.com 